\title{
Oliver Pye
}

\section{Für einen labour turn in der Umweltbewegung Umkämpfte Naturverhältnisse und Strategien sozial-ökologischer Transformation}

\section{Einleitung}

Kämpfe um die Inwertsetzung von Natur sind nicht neu. Der Kapitalismus als Globalsystem kann, Jason Moore (2015) folgend, als ständige Transformation des gesellschaftlichen Stoffwechsels mit der „Natur“ begriffen werden. Der krisenhafte, aber dynamische Drang zur Akkumulation führt dazu, dass immer neue Naturstoffe in Ressourcen verwandelt und angeeignet werden. So sind Konflikte ständige Begleiterscheinungen von neuen Einhegungen, Aneignungen und Akkumulationsregimen.

Dieser Beitrag versucht zunächst, Ansätze von David Harvey („Akkumulation durch Aneignung“), Jason Moore („world-ecology“) und Christoph Görg und Ulrich Brand („gesellschaftliche Naturverhältnisse“) aufeinander zu beziehen, um das Neue an der gegenwärtigen Regulation der globalisierten Naturverhältnisse zu skizzieren. Von Veränderungen am Kapuas Fluss in West-Kalimantan ausgehend, zeige ich, wie Landnahmeprozesse wie etwa die territoriale Ausdehnung der Palmölindustrie in globale Produktionsnetzwerke und einem corporate food regime (McMichael 2009) eingebettet sind. Anschließend charakterisiere ich die Kämpfe um die neuen Naturverhältnisse. Dabei werde ich Kämpfe gegen die „Akkumulation durch Enteignung"(Harvey 2003), etwa Kämpfe für kleinbäuerliche Landwirtschaft und Kämpfe gegen eine „Akkumulation durch Ausbeutung“ bzw. um die Bedingungen der Inklusion in neue Verwertungszusammenhänge (Arbeitskämpfe) gegenüberstellen.

Im dritten Teil beschäftige ich mich mit den Bewegungen, die über die Verallgemeinerung und Vernetzung von Kämpfen auf unterschiedliche Weise auf eine Transformation der gegenwärtigen Naturverhältnisse abzielen (Ernährungssouveränität, degrowth, buen vivir usw.). Der Beitrag diskutiert, inwieweit diese Bewegungen auf die Veränderungen in der Regulation der Naturverhältnisse adäquat reagieren. Ich argumentiere, dass die zurzeit entwickelten Strategien sozial-ökologischer Transformation vor allem die Verteidigungskämpfe gegen Einhegungen im globalen Süden (Ernährungssouveränität, indigene Landrechte) 
mit konsumkritischen Perspektiven im globalen Norden (degrowth, „imperiale Lebensweise") verknüpfen, ohne diese aber systematisch auf die sozialen Kämpfe innerhalb der Produktion zu beziehen.

Anders ausgedrückt finden die sozialen Kämpfe der prekarisierten ArbeiterInnen innerhalb der globalisierten Produktion keinen Eingang in die Strategien sozial-ökologischer Transformation. So bleiben auch internationale Netzwerke der Zivilgesellschaft an den Anfangs- und Endpunkten von globalen Produktionsketten der Naturverwertung stehen (Landkonflikte und Konsum von Palmöl, indigene Kämpfe gegen Bergbau und Handys), ohne sie transnational und radikal zu durchdringen. Dies erklärt die gegenwärtige Schwäche der Bewegungen für Umwelt- und Klimagerechtigkeit, eröffnet aber auch neue strategische Perspektiven. Wenn es keine sozial-ökologische Transformation mit Entfremdung geben kann, dann wird das dialektische Verhältnis zwischen kollektiven (Verteilungs-) Kämpfen und der Überwindung der Entfremdung zum entscheidenden Moment hin zu demokratischen gesellschaftlichen Naturverhältnissen.

\section{Globalisierte Naturverhältnisse}

Am Kapuas, dem längsten Fluss Indonesiens, wird Natur derzeit neu in Wert gesetzt. Der Fluss war für tausende von Jahren mit einer komplexen Landschaft aus Sumpfwäldern, Altarmen, Flutwäldern und saisonalen Seen Lebensraum für viele Fisch- und andere Tierarten. Die BewohnerInnen West-Kalimantans nutzten diese Biodiversität und den Fischreichtum für Jahrhunderte als Subsistenzgrundlage - und den Fluss als Handelsroute, um Waldprodukte, später dann Kautschuk, auf einem globalen Markt zu verkaufen. Diese sozial-ökologische Landschaft aus kleinbäuerlicher Landwirtschaft, agro-forstlichen Systemen, Wäldern und Fließgewässer entlang des Kapuas wird es so in naher Zukunft nicht mehr geben. Die gesamte Fläche der Provinz West-Kalimantan ist für Bergbau, monokulturelle Plantagenwirtschaft und Kohlenstoffspeicherung vorgesehen. Geplante REDD-Projekte für den Emissionshandel mit Kohlenstoff und Zellstoffplantagen umfassen über sechs Millionen Hektar, Holzkonzessionen knapp drei Millionen Hektar, Bergbaukonzessionen (z.B. für die Bauxitförderung) und Palmölmonokulturen jeweils fünf Millionen Hektar. In der Summe umfassen diese Vorhaben mit über 19 Millionen Hektar (mit Überlagerungen) mehr als die Gesamtfläche der Provinz (Radjawali u.a. 2017).

Es ist unschwer zu erkennen, dass in West-Kalimantan, wie in vielen anderen Gebieten Südostasiens (etwa Mindanao, West-Papua, Sarawak, Laos, Myanmar), ein Prozess der Landnahme, d.h. die „Einverleibung von nichtkapitalisierter Arbeitskraft und Erde“ (Dörre 2013: 114) stattfindet. Heute wird dieser Prozess als 
Fortsetzung der „ursprünglichen Akkumulation“ (Marx 1890. MEW 23: 741ff.) analysiert, oder, wie es David Harvey ausdrückt, als „Akkumulation durch Enteignung"(Harvey 2003: 144). Harvey (1981) argumentiert, dass die geografische Expansionsdynamik des Kapitals als „räumliche Lösung “ (spatialf fix) für die Problematik der Überakkumulation fungiert. Unternehmen erhöhen ihre Profitrate dadurch, dass sie immer neue Naturressourcen in Wert setzen und frisch "proletarisierte“ Arbeitskräfte ausbeuten (Harvey 1981: 8). Vermeintlich „nicht-kapitalistische“ Territorien werden vor diesem Hintergrund als neue Absatzmärkte, aber vor allem deshalb erobert, um Kapital Zugang zu „billigen Arbeitskräften, Rohstoffen und Land“ (ebd.: 139) zu ermöglichen. Diese „räumliche Lösung“ hat weitreichende Konsequenzen für die gesellschaftlichen Naturverhältnisse - nicht nur, weil die kapitalistische Ausbeutung von Naturressourcen ständig territorial expandiert (und somit auch ein räumlich ungleiches Nord-Süd-Verhältnis begründet), sondern auch, weil immer neue Naturstoffe (seltene Erden, genetische Informationen, Magnanknollen, gespeicherter Kohlenstoff usw.) kommodifiziert werden.

Auch für Jason Moore (2015: 17) ist der Zugang zu den „four cheaps“, d.h. billige Arbeitskraft, Nahrung, Energie und Ressourcen entscheidend für die Stabilität des Kapitalismus (vgl. Moore in PROKLA 185). Dem Ansatz des Weltsystems folgend, so Moore, war die Aneignung von Natur schon für die Entstehung des Kapitalismus im „langen 16. Jahrhundert“ (1450-1640) konstitutiv und seitdem immer als Teil des Verhältnisses zwischen Peripherie und Zentrum zu verstehen (world-ecology). In seinem Buch Capitalism and the Web of Life (2015) zeichnet Moore nach, wie die Inwertsetzung der ",unbezahlten Arbeit“ von „Natur, Kolonien und Frauen“ sich durch „ökologische Entwicklungskrisen“ (ebd.: 125) fortentwickelte. Diese nicht bezahlten Wertzuflüsse machten die Produktivitätszuwächse der Lohnarbeit in den stabilen Phasen kapitalistischer Entwicklung erst möglich - die „Dialektik aus Plünderung und Produktivität“ (Moore 2011: 43). Moore geht von einer historischen Ko-Produktion von Natur und Gesellschaft aus sowie davon, dass menschliches Handeln immer "gebündelt“ mit dem Rest der Natur stattfindet (2015: 29, 37). Kapitalismus ist daher nicht der Natur äußerlich, sondern selbst ein „ökologisches Regime“ und „Wall Street eine Weise, die Natur zu organisieren“ (Moore 2011).

In der deutschsprachigen Debatte ist dieser Gedankengang am ehesten beim Ansatz der gesellschaftlichen Naturverhältnisse zu finden, die Görg als „Gesamtheit der Praktiken, der institutionellen und organisatorischen Formen, in denen Gesellschaften ihre Verhältnisse zur Natur regulieren und transformieren " definiert (Görg 2004: 201). Görg greift auf die Frankfurter Schule zurück, die die „Nichtidentität“ von Natur (und Gesellschaft) betonte und kritisiert, dass der „Eigensinn“ der Natur ignoriert werde als fortsetzende Naturbeherrschung (Görg 2003: 42). Ulrich Brand und Christoph Görg verwenden für die Analyse gesellschaftlicher 
Naturverhältnisse regulationstheoretische Annahmen, bei denen relativ stabile Akkumulationsregime mit bestimmten „Regulationsweisen“ artikuliert werden (2003: 22). „Postfordistische“ Naturverhältnisse gründen sich demnach auf ein primär neoliberal geprägtes Akkumulationsregime, das durch eine Internationalisierung des Kapitals, globale Wertschöpfungsketten und neue Technologien im Bereich Transport, Information und Kommunikation geprägt ist. Damit verknüpft ist eine neue Regulationsweise, die an einem neoliberalen Wettbewerbsmodell orientiert ist und eine ,weitgehende Deregulierung der Kapital- und Finanzmärkte, verbunden mit einer umfassenden Privatisierung von öffentlichen Unternehmen, Versorgungseinrichtungen und sozialen Sicherungssystemen“ (ebd.: 23) vorantreibt. Die neue Regulationsweise strukturiert beispielsweise den Zugang zu genetischen Ressourcen über neue Abkommen wie die Konvention über biologische Vielfalt (CBD) und den Vertrag über geistiges Eigentum (TRIPS). Die Inwertsetzung von genetischen Ressourcen und die postfordistischen Naturverhältnisse insgesamt konzipieren Brand und Görg (2003: 219), ähnlich wie Harvey, als „die Nutzung nichtkapitalistischer Formen, die im Dienste der Inwertsetzung transformiert und kapitalistischen Gesellschaftsformen subsumiert werden“.

Diese Ansätze sind grundlegend für eine Analyse globalisierter Naturverhältnisse. Zusammen ergeben sie einen Analyserahmen, der die Aneignung und Verwertung von Natur als dynamisches Weltsystem begreift, bei dem ökologische Entwicklungskrisen (Moore 2015) neue Phasen stabilerer Akkumulationsregime hervorbringen, die durch neue Regulationsweisen (Görg/Brand 2003) gekennzeichnet sind. All dies lässt sich am Kapuas beobachten. Billige Natur (cheap nature) wird in Form von Ressourcen (Bauxit, Holz), Nahrung (Palmöl) und Energie (Agrodiesel, Kohle) angeeignet und global verwertet. Frisch proletarisierte Bäuerinnen, Bauern und MigrantInnen aus anderen Teilen Indonesiens dienen als billige Arbeitskräfte. Eine duale Lebensweise der Kleinbäuerinnen und -bauern, die auf einer Kombination von Subsistenz und Markt beruhte (Dove 2011), wird durch ein auf Kapital und Lohn reduziertes Verhältnis ersetzt. Verarmte und proletarisierte Bauern suchen ihr Glück, in dem sie im Fluss und entlang des Ufers nach Gold graben. Das Resultat ist die Beschleunigung der Doppelkrise aus Klimawandel (Torfentwässerung und Waldbrände) und Artenverlust (Umwandlung der Wälder und kleinbäuerlicher Landwirtschaft in Monokulturen).

Die Veränderungen in West-Kalimantan sind auf verschiedenen Raummaßstabsebenen mit globalen Prozessen verknüpft, im Falle der Palmölexpansion in die globalisierte Nahrungsmittelindustrie integriert. Dieser grundlegendste der gesellschaftlichen Stoffwechsel mit der Natur lässt sich in drei (der Idee der Akkumulationsregime analogen) relativ stabile „Nahrungsregime“ ( food regimes) unterteilen: in das „koloniale Nahrungsregime“, das „Entwicklungsnahrungsregime“ (1950er-1970er Jahre) und das heutige corporate food regime (McMichael 2009). 
„Billige Nahrungsmittel“ folgen dabei keineswegs dem Schema „Produktion im Süden für Konsumption im Norden“. Sie hängen vielmehr mit der Arbeitsproduktivität in der Landwirtschaft zusammen und haben zudem geopolitische Dimensionen. Im kolonialen Nahrungsregime diente vor allem Getreide aus den Siedlerstaaten (USA, Kanada und Australien) dazu, das Proletariat in den industrialisierten Zentren zu ernähren (ebd.: 141). Im zweiten food regime (eine Grundlage des fordistischen Akkumulationsregimes) war es die industrialisierte, kapital- und inputintensive und subventionierte Landwirtschaft Europas und der USA, die billige Überschüsse produzierte. Diese dienten dem Kalten Krieg, aber auch dazu, die Arbeitskosten im globalen Süden zu reduzieren.

Konstitutiv für das gegenwärtige corporate food regime (ebd.: 148), aber auch maßgeblich für globalisierte Naturverhältnisse insgesamt, ist die Herausbildung globaler Produktionsnetzwerke (Coe u.a. 2008; Dicken 2011). Aus der Kombination von kapitalintensiver, subventionierter Landwirtschaft im Norden und einer agro-industriellen ,grünen " Revolution im Süden, entstanden in den letzten Jahren Unternehmensnetzwerke, die nicht mehr eine bestimmte, nationale Landwirtschaft als Referenzpunkt haben, sondern räumlich globale Marketing- und Beschaffungsstrategien verfolgen. Weil Nahrungsmittel wie Mais, Soja, Palmöl oder Weizen zu flex crops (Borras u.a. 2014) mutieren und so als Grundstoff für die Energiewirtschaft, für die Chemieindustrie und für die Kosmetikindustrie dient, und weil die großfächige Monokulturproduktion auf Inputs wie gentechnisch veränderte Pflanzen, Pestiziden und Kunstdünger angewiesen ist, findet eine Fusion aus Agrarproduktion, Weiterverarbeitung, Chemie, Energie und Gentechnik statt. Ein Beispiel hierfür ist die Übernahme von Monsanto durch Bayer im Jahr 2017. Hinzu kommt die „Finanzialisierung des globalen Lebensmittelsystems" (Clapp 2014: 2). Ob bei der Finanzierung von land grabs, Finanzgeschäften mit Rohstofffonds oder Spekulationen mit Terminkontrakten auf Palmöl und Beimischungszertifikate: Hedge Fonds, Staats- und Pensionsfonds, Versicherungsunternehmen und Banken spielen eine immer wichtigere Rolle (Dietz u.a. 2016). Andere große Player im corporate food regime sind Supermarktketten, die nicht nur den Vertrieb kontrollieren, sondern mittlerweile auch direkt produzieren lassen. Das britische Unternehmen Hazelwood Foods, das Supermarktketten mit „own brand“- Fertigprodukten beliefert, hatte 2002 einen Umsatz von zwei Milliarden Euro und beschäftigte 7.000 ArbeiterInnen in 20 Fabriken in ganz Europa (Burch/Lawrence 2005: 8).

Wie Juan Infante Amate und Manuel González de Molina (2013) argumentieren, ist bei einer ökologischen Betrachtung der Landwirtschaft der gesamte Prozess agro-industrieller Produktion und Distribution zu berücksichtigen. Die Zone der territorialen Expansion (und Regenwaldumwandlung), die meist im Mittelpunkt der Analysen steht, ist verknüpft mit ausgedehnten sozial-ökologi- 
schen Landschaften von Plantagen und Mühlen, die den Lebens- und Arbeitsort vieler Millionen von ArbeiterInnen bilden. Diese wiederum sind über ein Transportsystem von Straßen und Lastwagen mit Raffinerien und Häfen verknüpft, die mittels Tanker mit anderen Häfen und mit, etwa in Deutschland, ansässigen Weiterverarbeitungs- und Fertigungsfabriken der Nahrungsmittelproduktion von Nestlé und Unilever, Stätten der Massentierhaltung in Niedersachsen, Chemiewerken von Bayer, Tausenden von Tankstellen und Agrodieselzapfstellen, Eisdielen und schließlich allen Supermarktfilialen des Landes verbunden sind. Jeder Knoten im globalen Produktionsnetzwerk ist selbst ökologisch eingebettet (Coe u.a. 2008: 278) und repräsentiert ein Schritt im (entfremdeten) Stoffwechsel mit Naturstoffen. Solche globalen Produktionsnetzwerke sind aber auch „Netzwerke verkörperter Arbeit“ (Cumbers u.a. 2008: 372), und die Knoten sind Alltagsorte der entfremdeten Arbeit von Kassiererinnen, Tankstellenwarten, Plantagenarbeiterinnen, Matrosen, Fabrikarbeiterinnen usw.

Bei Moore aber auch bei Brand, Görg und Wissen spielt die Lohnarbeit und speziell die ArbeiterInnenbewegung eine untergeordnete Rolle. Arbeit ist aber für das Verständnis kapitalistischer Naturverhältnisse zentral. Hier ist es hilfreich, die Arbeiten von John Bellamy Forster (Foster 2000; Foster u.a. 2010) und Paul Burkett (1999) hinzuzuziehen. Im Gegensatz zu Moore berufen sie sich auf die originäre Werttheorie bei Marx und dessen Unterscheidung zwischen Gebrauchswert und Tauschwert (Burkett 1999: 206). Statt einer einseitigen Beschränkung auf die „Akkumulation durch Enteignung “ betonen sie die intrinsisch naturzerstörerische Logik des Kapitals im „normalen“ Akkumulationsprozess. Dies liegt erstens darin begründet, dass Natur (neben Arbeit) zwar eine Quelle von Gebrauchswerten („des stofflichen Reichtums“, Marx 1890, MEW 23: 58) ist, aber nicht in die Wertbildung eingeht. Somit wird die Natur aus kapitalistischer Sicht „wertlos “ und damit der Beitrag der Natur zur Erzeugung von Gebrauchswerten systematisch entwertet (Burkett 1999: 73). Zweitens besteht (auch ohne Landnahme) ein grundlegender Widerspruch zwischen dem unendlichen Drang zur Akkumulation im Kapitalismus (G-W-G') und den natürlichen Grenzen der Erde.

Darüber hinaus führt die Entfremdung im Arbeitsprozess zu einer „Selbstentfremdung des Menschen von sich und der Natur" (Marx 1844, MEW 40: 519). Nicht nur das Produkt der Arbeit der LohnarbeiterIn ist ihr entäußert (das Kapital eignet es an), sondern auch ihre Arbeit selbst, ihre „Lebenstätigkeit“ (ebd.: 517). In anderen Worten, das, was uns zu Menschen macht, unser „Gattungscharakter" (ebd.: 517), wird uns entfremdet und damit auch das praktische Verhältnis (gesellschaftlicher Stoffwechsel) mit der Natur. Aus diesen drei Widersprüchen entwickeln Foster u.a. (2010) den Begriff des ecological rift, eines Bruchs im gesellschaftlichen Stoffwechsel mit der Natur. Was Marx zunächst als Erklärung der Erschöpfung der Böden in der kapitalistischen Landwirtschaft entwickelte, 
erweitern die AutorInnen mit Blick auf die planetarischen Grenzen (Klimawandel, Artensterben, Stickstoffkreislauf, Versauerung der Meere usw.) und erklären die großen ökologischen Krisen unserer Zeit aus der Akkumulationsdynamik der kapitalistischen Produktionsweise.

\section{Umkämpfte Naturverhältnisse}

Wenn wir Naturverhältnisse nicht nur als die Aneignung noch nicht verwerteter Natur, nicht nur als Aneignung von Ressourcen und Wissen „nicht-kapitalistischer Gesellschaften"verstehen, sondern als sozial-materielles Verhältnis, das jeden Schritt im gesellschaftlichen Stoffwechsel mit der Natur kennzeichnet, dann hat dies auch Konsequenzen für unser Verständnis von Kämpfen. Wenn wir davon ausgehen, dass Naturverhältnisse nicht nur an der territorialen Grenze, d.h. in der Mine oder an der Waldgrenze produziert werden, sondern entlang der gesamten Produktionskette, dann sind Kämpfe entlang dieser Kette miteinander verknüpft und für einen Wandel bestehender Naturverhältnisse relevant: Die Kämpfe gegen die Akkumulation durch Enteignung müssen zu Kämpfen gegen Akkumulation durch Ausbeutung in Beziehung gesetzt werden. Auch Harvey fordert dies ein. Weil „beide Formen des Kampfes in der historischen Geographie des Kapitalismus organisch verknüpft sind“ (2003: 181), müsse die globalisierungskritische Bewegung, die v.a. aus Kämpfen gegen Akkumulation durch Enteignung hervorgeht, die „dialektische Beziehung zu den Kämpfen im Feld der erweiterten Reproduktion" anerkennen. Diese Verbindungen werden in den aktuellen Diskussionen um Kämpfe um Land oder Bergbau nur in seltenen Fällen vollzogen.

Akkumulation durch Enteignung wird von einer Vielzahl von Kämpfen begleitet, die an der Grenze der territorialen Expansion, an der physisch-materiellen Landnahme selbst verortet sind: Kleinbäuerinnen und -bauern wehren sich gegen land grabbing oder eine agro-industrielle Umstrukturierung der Landwirtschaft, Indigene verteidigen kommunales Eigentum gegen die Privatisierung von Land und Wissen, KleinfischerInnen versuchen, Großstaudämme und die Zerstörung ihrer auf Flussökosysteme angewiesenen Lebensweise zu verhindern. Auch am Kapuas finden solche Kämpfe statt. Gegen die immer weiter expandierende Palmölindustrie setzen sich Kleinbäuerinnen und -bauern und indigene Gruppen in Netzwerken wie AMAN (Aliansi Masyarakat Adat Nusantara - Allianz Indigener Völker des Archipels) oder FAMKI (Front Aliansi Masyarakat Korban Investasi - Vereinigung der Opfer von Investitionen) zur Wehr. Sie lehnen Palmölprojekte in ihrem Dorf ab, schreiben Petitionen, demonstrieren oder besetzen Straßen und Büros. Indigene versuchen, ihr Territorium mit Drohnen zu kartieren, um ihre Rechte auf Waldgebieten oder Überschreitungen durch Bergbauunternehmen zu dokumentieren 
(Radjawali u.a. 2017). Eine „Zivilgesellschaftliche Allianz für eine gerechte und nachhaltige Raumplanung "(Koalisi Masyarakat Sipil untuk Tata Ruang yang Adil dan Berkelanjutan) hat erfolgreich in die Landnutzungsplanung der Provinz interveniert, um mehr Mitsprache seitens der betroffenen Dörfer zu erwirken (ebd.).

Diese Aneignungen können zwar nicht wirklich als Eroberung „nicht-kapitalistischer" Räume bezeichnet werden - dafür waren die Kleinbäuerinnen und -bauern und Indigenen um den Kapuas zu lange schon in globalen Märkten integriert. Doch die Kämpfe sind durchaus eine Verteidigung einer gewissen kleinbäuerlichen Autonomie, einer Lebensweise, die auf einer dualen Ökonomie aus Subsistenz und Marktproduktion basiert, gegenüber der Durchkapitalisierung durch agroindustrielle und extraktivistische Unternehmen.

Solche Kämpfe gibt es nicht nur am Kapuas, sondern sie haben Asiens beschleunigte Modernisierung und Landnahme insgesamt begleitet: Die großen AntiStaudammbewegungen in Narmada (Indien), gegen den Dreischluchtendamm in China oder am Pak Mun in Thailand; die Kämpfe der Penan (Malaysia) und der Chipkobewegung (Indien) gegen Abholzung; die Proteste von KleinfischerInnen gegen Trawler in der Straße von Malakka; die zivile Ungehorsamkeitsbewegung von thailändischen Kleinbauern gegen Vertreibungen durch das industrielle Zellstoffplantagenprojekt Khor Jor Kor (Pye 2005) und das Forum der Armen in Thailand, das verschiedene Kämpfe gegen die Akkumulation durch Enteignung vereinte (Missingham 2003); die Allianz zwischen indigenen und kirchlichen Gruppen gegen Bergbau in den Philippinen, der Kampf der Samin gegen Heidelberg Cement auf Java; die Landbesetzungen von La Via Campesina - die Liste ließe sich fortführen.

Diese Kämpfe sind integrales Moment konfliktreicher Landnahme: Hier wehren sich Menschen gegen Einhegungen, die Teil der kapitalistischen Modernisierung Asiens sind. Komplizierter wird es bei den Kämpfen, bei denen es um die Bedingungen der Inklusion in diese neue Verwertungszusammenhänge geht. Im Palmölbereich sind diese verbreiteter als die prinzipielle Ablehnung einer neuen Plantage. Weil Unternehmen die Zustimmung der lokalen LandeigentümerInnen (auch der traditionellen) einholen müssen, entstehen viele Konflikte um die genauen Konditionen dieser Inklusion: Wie viel Land geben die lokalen Bäuerinnen und Bauern ab, wie viel Palmölfläche erhalten sie im Gegenzug; wie hoch sind die Schulden, die sie für die Anlage der Palmölparzelle abbezahlen müssen; welchen Anteil bekommen sie von den Einnahmen der Plantage und so weiter. Oft klaffen Versprechungen und reale Praxis auseinander. Dann kommt es zu Sabotageakten, Diebstahl an Palmölfrüchten und Protestaktionen. In Ketapang, West-Kalimantan ist etwa im Zuge der Finanzkrise 2008 eine Palmölfirma bankrottgegangen. Die Palmölmühle stand still. Die KleinproduzentInnen organisierten Protestmärsche und gingen vor Gericht, weil sie ihre Früchte nicht verkaufen konnten, und ihren Landparzellen deshalb zu verlieren drohten (Interviews mit Kleinprodu- 
zentInnen, partizipative Beobachtung, 2008). Ein Kampf gegen Akkumulation durch Enteignung war das nicht. Und wie sollen wir die Zehntausende von GoldschürferInnen einordnen, die entlang der Kapuas einen immensen ökologischen Schaden anrichten? Auch ihr Kampf - z.B. um Entkriminalisierung - gehört zu den gesellschaftlichen Naturverhältnissen, auch wenn er keine Verteidigung einer prä-kapitalistischen oder umweltschonenden Lebensweise repräsentiert.

Wenn wir aus einer Perspektive globalisierter Naturverhältnisse nicht nur die Ränder, sondern die gesamte Kette der Palmölindustrie betrachten, von der Plantage bis zur Supermarktfiliale, dann bekommen die Kämpfe der ArbeiterInnen einen anderen Stellenwert in der Betrachtung von umkämpften Naturverhältnissen. An anderer Stelle habe ich nachgezeichnet, wie die Alltagspraxis der indonesischen MigrantInnen einen umkämpften, sozial-ökologischen Raum zwischen Indonesien und Malaysia produziert (Pye 2013). Sie nutzen ihre sozialen Netzwerke, um ein repressiv reguliertes Arbeitsregime zu umgehen und herauszufordern. So ignorieren sie Einwanderungsbestimmungen, um Grenzen zu überqueren oder um Familien nachzuführen. Sie setzen höhere Löhne durch, indem sie trotz Verbot ihren Arbeitsplatz wechseln. Sie führen wilde Streiks durch und bringen so die Palmölproduktion zum Stillstand. Dabei geht es immer um Löhne und um die soziale Reproduktion - die Frage nach Familienzusammenführung, Erziehung der Kinder, Pflege der Alten und Kranken spielt eine entscheidende Rolle in den Lebensentwürfen der MigrantInnen (Pye u.a. 2012). Es geht aber immer auch, an jeder Stelle im Produktionsprozess, um die Entfremdung zur Arbeit und zur Natur.

In Asien stellen die Kämpfe der ArbeiterInnen vom Ausmaß her alle Kämpfe gegen Aneignungen in den Schatten. Ein Beispiel ist die über sechs Jahre fast durchgehende Welle von Streiks in den Jahren 2006 bis 2011 in Vietnam. Die Zahl der Streiks stieg 2006 von 150 auf 400, um 2011 fast 900 zu erreichen. Die Arbeitskämpfe fanden vor allem in ausländischen Unternehmen der globalen Produktionsnetzwerke (Schuh- und, Textilproduktion) statt und drehten sich vornehmlich um die Erhöhung von Löhnen. Die Verdopplung des Mindestlohns in Vietnam in diesem Zeitraum geht auf diese Streikbewegung zurück (Siu/Chan 2015). Die mehrheitlich weiblichen TextilarbeiterInnen in Kambodscha haben ähnlich erfolgreich 2014 mit einer Streikbewegung, die Phnom Penh lahmlegte, den Mindestlohn auf 100 US-Dollar fast verdoppelt. In China wuchs die Anzahl von Arbeitskonflikten von 40.000 im Jahr 2000 auf über 400.000 in 2010, als es zu einer Eskalation von Kämpfen im Automobilsektor kam (Butollo/ten Brink 2012: 422). Auch hier ging es um eine Kombination aus Lohnforderungen und die Einforderung von bestehenden ArbeiterInnenrechten und auch hier konnten ArbeiterInnen Lohnzuwächse von 30 Prozent durchsetzen (ebd.: 428).

Nicht nur das Ausmaß der neuen Arbeiterbewegungen, sondern auch ihre Militanz ist bedeutsam. Es ist erstaunlich, dass in autoritären Regimen wie China, 
Kambodscha und Vietnam über Wochen oder Monate ArbeiterInnen aus verschiedenen Fabriken wild streiken. Die Streiks in China zeichnen sich dadurch aus, dass sich ArbeiterInnen massenhaft und aktiv daran beteiligen und die Wahl von Streikkomitees an den offiziellen („gelben“ oder parteitreuen) Gewerkschaften vorbei geht (Butollo/ten Brink 2012: 432). Apo Leong (2017: 43) spricht von einer neuen Normalität, in der eine neue Generation von ArbeiterführerInnen und organisers heranwächst. Benny Juliawan (2011:361) berichtet von der militanten Praxis des „Fegens” in Indonesien, ähnlich jener der fliegenden Streikposten, wo demonstrierende ArbeiterInnen in andere Fabriken (die zunächst nichts mit dem Streik zu tun haben) hineingehen und die Fabrikleitung zwingen, ihre ArbeiterInnen am Protestzug teilnehmen zu lassen. Die Streikenden konnten so hunderte von Fabriken in ihren Kampf hineinziehen. Diese militanten Aktionen weiteten sich zwischen 2011 und 2013 zu einer Bewegung gegen ein Gesetz zur Legalisierung von temporären Beschäftigungsverhältnissen aus, an der sich über sechs Millionen ArbeiterInnen in zwei Generalstreiks beteiligten. Auch hier praktizierten Streikende das „Fegen” anderer Fabriken; hinzu kamen Blockaden von Mautstraßen und die Besetzung ganzer Industriegebiete (Mufakhir 2017: 262).

Ein Merkmal der neuen ArbeiterInnenbewegung in Asien ist ihre zunehmende Ausweitung über direkte Arbeitskämpfe hinaus. 2016 nahmen bis zu 180 Millionen ArbeiterInnen an dem „wahrscheinlich größten Generalstreik der 'Weltgeschichte" (Chattopadhyay/Marik 2016: 1) in Indien teil. Sie protestierten gegen die Privatisierungspolitik der Regierung Modis, gegen prekäre Beschäftigung und für höhere Löhne, eine allgemeine Gesundheitsversorgung und soziale Sicherung für alle.

\section{Strategien sozial-ökologischer Transformation}

Aus der Verallgemeinerung von Kämpfen um Naturverhältnisse entstehen Strategien sozial-ökologischer Transformation. Der im deutschsprachigen Raum zurzeit einflussreichste Versuch, diese Ansätze zusammenzudenken und für eine umfassende Perspektive eines „radikalen Reformismus“ zu werben, ist das Buch Die Imperiale Lebensweise von Ulrich Brand und Markus Wissen. Für die Autoren geht es mit dem Begriff der Transformation nicht um eine ökologische Modernisierung des Kapitalismus, sondern um eine radikale Neugestaltung gesellschaftlicher Naturverhältnisse und eine grundlegende Veränderung von „Produktions-, Distributions- und Konsumnormen." Sie diskutieren eine Vielzahl von Ansätzen, die derzeit auf die Entwicklung alternativer Alltagspraktiken und die Intervention in die Regulation von Naturverhältnissen auf verschiedenen Ebenen abzielen. 
Strategien wie Ernährungssouveränität, buen vivir und degrowth lassen sich als Ausweitungen von Kämpfen gegen Akkumulation durch Enteignung verstehen. Ernährungssouveränität, von der 1993 gegründeten internationalen Bewegung von Kleinbauern und Landarbeitern La Via Campesina entwickelt, ist sowohl eine Verteidigung einer kleinbäuerlichen Landwirtschaft gegen die Aneignung durch Agrobusinesskonzerne als auch ein Entwurf einer alternativen Produktions- und Konsumweise. In den Grundsätzen einer arbeitsintensiven und kleinflächigen Bearbeitung des Bodens, eines Nährstoffkreislaufs (organische Dünger, Aufbau der Humusschicht usw.) und regionaler Produktions- und Vertriebssysteme werden Konturen einer Landwirtschaft deutlich, die eher nach Gebrauchswerten als nach Tauschwerten organisiert ist. Buen vivir - gut Leben - ist darüber hinaus ein philosophischer Ausdruck von Kämpfen indigener Völker etwa in Ecuador, Bolivien, Peru oder Chile gegen eine auf Extraktivismus beruhende Entwicklungsstrategie. Seit 2008 in der ecuadorianischen Verfassung verankert, wird hier eine neue Beziehung mit der Natur angestrebt, die sich auf eigenständige Rechte von „Mutter Erde“ (madre tierra) gründet (Caria/Dominguez 2016). Degrowth ist eine vor allem in Europa entstandene Bewegung, die von einer Wachstumskritik ausgehend die unverhältnismäßige Aneignung von Ressourcen im globalen Süden infrage stellt. Eine Vielzahl von Praktiken und Lebensweisen wie „Radfahren, Wiederverwertung, Vegetarismus und Veganismus, Wohnprojekte, ökologische Landwirtschaft, Ökodörfer, solidarische Ökonomie, Konsumentenkooperativen, alternative (oder ethische) Banken und Kreditkooperativen, dezentralisierte Energiekooperativen " (Demaria u.a. 2013: 202) wird mit gesellschaftlichen Perspektiven von Umverteilung und Gerechtigkeit verknüpft, die auf eine Reduktion des Ressourcenverbrauchs und Energiedurchflüssen abzielen.

In allen drei Ansätzen werden Konturen anderer Naturverhältnisse deutlich, die wichtige Impulse für eine sozial-ökologische Transformation liefern. Gleichzeitig repräsentieren sie auch die Schwächen bisheriger Ansätze. Sie setzen alle an den Rändern der Produktion an - an den Grenzen der Aneignung und an den Konsumgütern - ohne das Innere der globalen Produktionsnetzwerke zu durchdringen (vgl. Max Koch in diesem Heft). So kann Ernährungssouveränität eine kleinbäuerliche Landwirtschaft gegenüber der ständigen Expansion kapitalistischer Monokulturen verteidigen, aber nicht aufzeigen, wie das corporate food regime konkret infrage gestellt, aufgehalten und sozial-ökologisch transformiert werden kann. Buen vivir kann das Prinzip der Rechte der „Mutter Erde“ formulieren, aber alleine eine globale Extraktivismus-Industrie nicht transformieren und ersetzen. Auch die degrowth-Bewegung kann mit weniger Konsum und lokalen Produktionsnetzwerken den permanenten Zwang zur Akkumulation (und damit auch die Akkumulation durch Enteignung) nicht außer Kraft setzen. 
Wie Foster u.a. (2010: 395) argumentieren, ist eine neue „Ökologie des Konsums“ nur als Teil einer neuen „Ökologie der Produktion“ möglich. Ein Blick zurück auf das corporate food regime und das Ziel einer Transformation in Richtung Vegetarismus verdeutlicht das Dilemma: Unsere Nahrungsmittelproduktion wird in globalen Produktionsnetzwerken organisiert, die agro-industrielle Landwirtschaft mit einer globalen Logistikinfrastruktur, Supermarktketten und Finanzinstitutionen verknüpft. Der „transnationale Tierproteinkomplex“ verbindet dabei Soja- und Palmölmonokulturen in Brasilien und Südostasien mit subventionierter Weizen- und Maisproduktion in den USA und hoch industrialisierte Mastbetrieben in Deutschland (McMichael 2009: 141). Eine sozial-ökologische Transformation muss an der Auflösung dieses Produktionssystems ansetzen und auf eine dezentralisierte sowie tiergerechte Tierhaltung mit überwiegend vegetarischer Ernährungsweise zielen. Aus einer konsumkritischen Perspektive zeichnen sich bereits Veränderungen ab. Die Anzahl der VeganerInnen in Deutschland ist von 80.000 im Jahr 2008 auf heute 1,3 Millionen angewachsen, die Anzahl der VegetarierInnen hat sich in den letzten 20 Jahren verzehnfacht (Vebu, ohne Datum). Gleichzeitig ist die Fleischproduktion in Deutschland stetig angewachsen und hat 2016 mit über acht Millionen Tonnen einen Höchstwert erreicht (HBS/BUND 2016). Der Anstieg der Zahl von VegetarierInnen (bzw. die Höhe des Fleischkonsums) tangiert die Akkumulation der Fleischindustrie nicht - immer größere Massentierbetriebe, immer kapitalintensivere Schlacht- und Verarbeitungsmethoden, immer mehr Überproduktion (ca. ein Drittel) und immer „billigeres“ Fleisch sind die Folge.

Eine vegetarische Ernährungsweise und die Bewegung der VeganerInnen sind ungemein wichtig, um eine alternative Praxis zu entwickeln und gesellschaftlich gegenhegemonial zu wirken. Ebenso wichtig sind Ansätze wie Ernährungssouveränität oder degrowth, weil sie eine prinzipielle Kritik an kapitalistischen Naturverhältnissen formulieren und eine Alternative sichtbar machen. Um aber wirksam zu werden, müssen sie mit einer Transformationsstrategie verknüpft werden, die Arbeit und die ArbeiterInnenbewegung ins Zentrum stellt. Eine degrowth-Perspektive kann sich nicht auf eine „ökologisch bewusste globale Mittelschicht“ beschränken, sondern muss einen „Dialog mit einer breit definierten, globalen Arbeiterklasse“ aufnehmen, auch mit jenen Millionen, die in den fossilistischen Industrien arbeiten (Barca 2017). Eine strategische Re-Orientierung auf Arbeit, Produktion und ArbeiterInnenbewegung ist, wie im Folgenden dargelegt wird, aus drei Gründen sinnvoll: 1. die zentrale Problematik der Entfremdung, 2. die Bedeutung von Lohnkämpfen und 3. das Potenzial des Proletariats als universaler Klasse.

Die zahlenmäßige Bedeutung des Proletariats im corporate food regime, wie in den neuen Palmöllandschaften, ist unbestreitbar. Millionen von ehemaligen Kleinbäuerinnen und -bauern oder Landlosen sind zu Lohnabhängigen auf den Palmölplantagen und in den Mühlen geworden. Das tragische Dilemma ist, dass 
sie in ihrer täglichen Arbeit dieses entfremdete Verhältnis zur Natur produzieren. Die ArbeiterInnen roden den Wald, legen die neuen Plantagen an, wenden Herbizide und Kunstdünger an, ernten die Ölpalmenfrüchte, transportieren diese in die Mühle und pressen dort das Öl aus. An jedem dieser Schritte agieren sie im Stoffwechsel mit der (nicht menschlichen) Natur. Doch die ArbeiterInnen verrichten diese Arbeit nur um des Lohnes willen. Sie verkaufen ihre Arbeitskraft - und damit die Gestaltung ihres Verhältnisses mit der Natur. Sie werden so von ihrer „Lebenstätigkeit“ entfremdet und somit auch von dem täglichen Stoffwechsel mit der Natur. Was sie der Natur antun, entscheidet der Vorarbeiter, die Managerin und der Kapitalist, somit schließlich die Konkurrenz und der blinde Zwang zur Akkumulation. Dies verknüpft die Umwandlung von kleinbäuerlicher Landwirtschaft und Regenwäldern zu Palmölplantagen mit der entfremdeten Tätigkeit an allen weiteren Knoten im gesellschaftlichen Stoffwechsel, bis es schließlich in Form von (entfremdeten) Produkten in den Supermärkten landet. Eine Transformation in Richtung demokratischer Gestaltung der gesellschaftlichen Naturverhältnisse muss diesen entfremdeten Kern im gesamten globalen Produktionsnetzwerk aufheben.

Wenn sie in ihren biographischen Erzählungen über die Arbeit sprachen, ging es ArbeiterInnen der Palmölindustrie fast immer um den Lohn (biografische Interviews mit PalmölarbeiterInnen 2010-2011). Die konkrete Tätigkeit, ob sie nun „Gift“ versprühen oder eine Maschine in der Palmölmühle führen, wurde anteilslos erduldet und nur beiläufig erzählt. Stattdessen diskutierten sie intensiv den Stundenlohn, Zulagen, Produktivitätsschübe seitens des Managements, unterschiedliche Lohnniveaus und Lohnerhöhungen der letzten zehn Jahre. Genau dies drückt die Entfremdung aus - und einen scheinbaren Widerspruch zwischen dem Proletariat und dem Ziel einer nachhaltigen Gestaltung von Naturverhältnissen. Denn es sind die ArbeiterInnen, die die Beherrschung über die Natur konkret umsetzen, die Akkumulation durch Enteignung ermöglichen und den Wachstumsdrang mit Lohnerhöhungen (und damit mehr Konsum) anheizen. Und doch sind die Kämpfe für mehr Lohn der Schlüssel zur Überwindung der Entfremdung und zur Herstellung demokratischer Naturverhältnisse.

Obwohl auf einem vergleichsweise noch niedrigen Niveau, entzünden sich kleinere kollektive Protestaktionen und einzelne wilde Streiks meistens am Lohn. Weil er der einzige Grund für die Arbeit in den Plantagen darstellt, ist seine Höhe von entscheidendem Interesse für die ArbeiterInnen. Welche Lohnhöhe sie als angemessen empfinden, hängt davon ab, wie viel sie zur längerfristigen sozialen Reproduktion der gesamten Familie als notwendig erachten. An der Lohnfrage werden damit zwei grundsätzliche Widersprüche deutlich. Zum einen geht es um die Mehrwertrate, also darum, wie sehr die ArbeiterInnen ausgebeutet werden und wie schnell die Akkumulation des Mehrwerts vonstattengeht. Damit geht es aber auch um den Widerspruch zwischen Gebrauchswert (die soziale 
Reproduktion der ArbeiterInnen) und Tauschwert (zur weiteren Akkumulation). Kommt es zum Streik, dann kommt es zu zwei qualitativen Veränderungen. Erstens wird der Prozess der Akkumulation sabotiert, und zweitens überwinden die ArbeiterInnen für die Dauer des Streiks ihre Entfremdung: Sie verkaufen nicht mehr ihre Arbeitskraft, sondern setzen diese bewusst anders ein (z.B. in die Organisation des Streiks).

Lohnkämpfe und Streiks können nur kurzfristig die Logik der Akkumulation und der Entfremdung durchbrechen. Längerfristig dienen sie aber dazu, dass kollektive Arbeiterorganisationen entstehen, welche die Grundlage für einen „Verein freier Menschen“ sind, die „mit gemeinschaftlichen Produktionsmitteln arbeiten und ihre vielen individuellen Arbeitskräfte selbstbewusst als eine gesellschaftliche Arbeitskraft verausgaben“ (Marx 1890, MEW 23: 92). Eine demokratische und selbstbestimmte Produktion für Gebrauchswerte ermöglicht wiederum ein prinzipiell anderes Verhältnis zur Natur (Burkett 1999: 225ff; Foster 2000). Wie Stefania Barca (2017) schreibt, wird die Bedeutung einer EntEntfremdung (de-alienation) für eine degrowth Strategie an der Wiederaneignung von Arbeit deutlich. In diesen Momenten kommt es zu einem Umweltschutz der ArbeiterInnenklasse (working class environmentalism) (Barca 2012) und zu Versuchen, den gesellschaftlichen Stoffwechsel mit der Natur emanzipatorisch zu organisieren. Barca (2017) nennt in diesem Zusammenhang die Gewerkschaft der Kautschukzapfer im Amazonas, die den Regenwald als Ort ihrer sozialen Reproduktion verteidigten und die Ri-Maflow-Fabrik in Italien, die 2009 von ArbeiterInnen besetzt und übernommen wurde. Letztere hörten auf, Autoteile zu produzieren und errichteten stattdessen unter dem Motto „reuse, re-cycle, re-appropriate" ein Zentrum für Gerätereparatur und für die Verarbeitung von lokalen landwirtschaftlichen Produkten.

Ein inspirierendes Beispiel eines solchen working class environmentalism sind die grünen Blockaden (green bans) der Baugewerkschaft von New South Wales, Australien in den 1970er Jahren. Eigentlich müssten BaugewerkschafterInnen, wenn sie einer Pro-Wachstums-Linie (= mehr „Arbeitsplätze“) ihrer eigenen Unternehmen folgen, kritiklos für eine Expansion der Bauindustrie eintreten. Doch zwischen 1971 und 1975 verhinderte die Gewerkschaft (zusammen mit BürgerInneninitiativen) Bauprojekte, die sie als umweltpolitisch und städteplanerisch schädlich einstuften. Die ArbeiterInnen weigerten sich, historische Gebäude oder Wohnhäuser zu demolieren, Baumaßnahmen in Parks und Naturschutzgebieten durchzuführen oder Stadtautobahnen zu bauen (Burgman/Burgman 1999: 53). Gewerkschaftsführer Jack Mundey vertrat offensiv die Position, dass BauarbeiterInnen das Recht hätten, ihre Arbeit nur für gesellschaftliche sinnvolle und verantwortungsvolle Aufgaben einzusetzen (ebd.: 53). Dies entsprach einer Gewerkschaftsphilosophie der „universellen Klasse“, bei der die Gesamtinteressen 
der ArbeiterInnenklasse (wie eine intakte Umwelt) und nicht nur Partikularinteressen verfolgt werden (ebd.).

Dass Gewerkschaften oder ArbeiterInnen sich aktiv an der Transformation oder gar Abschaffung ,ihrer" Industrie beteiligen, ist für manche sicherlich schwer vorstellbar. Zu stark scheint der vermeintliche Interessengegensatz zwischen ArbeiterInnenbewegung und Umweltbewegung zu sein, wie die Demonstrationen von RWE-MitarbeiterInnen für Kohle, oder die Positionierung der IGBCE für Atomkraft nahelegen. Doch auch in der heutigen Bewegung für Klimagerechtigkeit entstehen Möglichkeiten für eine Reorientierung auf die ArbeiterInnenbewegung. In Südafrika hat die Kampagne „One Million Climate Jobs“ eine erfolgreiche Allianz zwischen Teilen der ArbeiterInnenbewegung und der Klimagerechtigkeitsbewegung ermöglicht. Forderungen nach öffentlich finanzierten Jobs im Bereich erneuerbaren Energien oder öffentliche Verkehrssysteme werden gegen eine Agenda der grünen Modernisierung formuliert und mit Energiedemokratie und Ernährungssouveränität verknüpft (Barca 2017). In Großbritannien genießt eine ähnliche Kampagne die Unterstützung etlicher Gewerkschaften ${ }^{1}$. Auf der diesjährigen Konferenz des Trade Union Congress wurde eine Resolution verabschiedet, die einen "gerechten Übergang" in eine nicht-fossilistische Energieproduktion fordert, inklusive der Rückkehr der Energieproduktion in die öffentliche Hand, das Ende von Investitionen von Pensionsfonds in fossile Industrien und die Umstellung auf regenerative Energien.

\section{Schlussfolgerungen}

Die Landnahme der letzten Jahrzehnte und die Expansion kapitalistischer Naturverhältnisse in Regionen wie Südostasien haben zu einer Vielzahl von Kämpfen geführt, die eine antikapitalistische Perspektive für eine sozial-ökologische Transformation inspiriert haben. Eine Ausweitung dieser Kämpfe und Strategien führte bisher zu einer Kombination, die alternative Umgangsformen mit der Natur im globalen Süden (Ernährungssouveränität, buen vivir) mit einer konsumkritischen Haltung (degrowth, imperiale Lebensweise) im globalen Norden verbindet. Diese Ansätze sind sowohl für die Kritik an der kapitalistischen Beherrschung der Natur als auch für die Entwicklung einer Praxis der Alternativen ungemein wichtig. Die Praxis kapitalistischer Produktion, das prinzipielle Pro-

1 Bakers, Food and Allied Workers Union (BFAWU), Communication Workers Union (CWU), Fire Brigades Union (FBU), National Union of Students (NUS), Public and Commercial Services Union (PCS), Transport Salaried Staffs Association (TSSA), Unite, the union (UNITE), and the University and College Union (UCU). 
blem der Entfremdung in den herrschenden Naturverhältnissen sowie die Rolle der ArbeiterInnenbewegung in Strategien sozial-ökologischer Transformation werden dabei aber vernachlässigt.

Die ArbeiterInnenklasse und die ArbeiterInnenbewegung spielen in diesen Perspektiven auch deshalb keine Rolle, weil die ökologische Krise als eine Art geografisch-territoriales Verteilungsproblem analysiert wird. In der degrowthErklärung von 2010 wird die ökologische Krise auf das Konsumverhalten einer „internationalen Elite und einer globalen Mittelschicht“ zurückgeführt. Deren „Konsummuster“ - und ein Statusdenken, das durch „die Akkumulation von materiellen Gütern“ befriedigt wird - würden ökologische und soziale Schäden anrichten, vor allem deshalb, weil sie von anderen "nachgeahmt werden“ (Degrowth Declaration 2010). Das Problem ökologischer Zerstörung wird damit auf eine Verteilungsfrage von Konsum reduziert. Ähnlich argumentieren Ulrich Brand und Markus Wissen (2017: 12) in ihrer Darstellung einer „imperialen Lebensweise“ im globalen Norden, die auf der Aneignung von „Natur und Arbeitskraft“ im globalen Süden beruhen soll.

Die von Brand und Wissen vorgebrachte Strategie einer „Deprivilegierung“ "großer Teile der Lohnabhängigen“"(2017: 52), d.h. ein Absenken des materiellen Lebensstandards im globalen Norden ist eine Sackgasse, die keine Strahlkraft über die von ihnen bedienten Milieus hinaus besitzen kann. Eine Erklärung, die „zu viel Konsum“ im globalen Norden als Ursache für die ökologischen Krisen unserer Zeit identifiziert, führt zu einer politischen Praxis, bei der die Verringerung des eigenen ,ökologischen Fußabdrucks“ im Zentrum steht (keinen SUV kaufen, vgl. ebd.: 125ff.; Dörre 2013: 131). Die Frage der Produktion und wie diese transformiert werden kann, wird damit umschifft (Foster 2011). Eine aus der Konsumkritik entwickelte Perspektive kann vor allem kein produktives Verhältnis zu der überwiegenden Mehrheit der sozialen Kämpfe - den Lohnkämpfen - entwickeln.

Eine sozial-ökologische Transformation kapitalistischer Naturverhältnisse wird nur mit der aktiven Beteiligung der Lohnabhängigen gelingen. Lohnkämpfe müssen deswegen als ein wichtiger Ausgangspunkt betrachtet werden, weil hier sowohl die Akkumulation unterbrochen als auch die Entfremdung infrage gestellt werden kann. In dieser Wiederaneignung der Arbeit, unserer power-to-do (Holloway 2005), liegt der Schlüssel zur demokratischen Gestaltung der gesellschaftlichen Naturverhältnisse. Entscheidend wird sein, ob die ArbeiterInnenbewegung als universelle Klasse agiert und konsequent für die Transformation von Naturverhältnissen eintritt. Dafür müsste sich die auf Systemveränderung ausgerichtete Umweltgerechtigkeitsbewegung aber auch als Teil dieser universellen Klasse verstehen. Ein labour turn der Umweltbewegung muss her. 


\section{Literatur}

Amate, Juan Infante/González de Molina, Manuel (2013): 'Sustainable de-growth' in agriculture and food: an agro-ecological perspective on Spain's agri-food system (year 2000). In: Journal of Cleaner Production 38: 27-35.

Barca, Stefania (2012): On working-class environmentalism: a historical and transnational overview. In: Interface: a journal for and about social movements 4(2): 61-80.

- (2017): The labor(s) of degrowth. URL: https://entitleblog.org/2017/01/31/the-labors-ofdegrowth/, Zugriff: 4.11.2017.

Borras, Saturnino M.Jr./Franco, Jennifer C./Isakson, Ryan/Levidow, Les/Vervest, Pietje (2014): 'Towards Understanding the Politics of FlexCrops andCommodities: Implications for Research and PolicyAdvocacy'. In: Think Piece Series on Flex Crops and Commodities (1). Amsterdam.

Brand, Ulrich/Görg, Christoph (2003): Konflikte um genetische Ressourcen und die Internationalisierung des Staates. Münster.

-/Wissen, Markus (2017): Imperiale Lebensweise. Zur Ausbeutung von Mensch und Natur im globalen Kapitalismus. München.

Burch, David/Lawrence, Geoffrey (2005): Supermarket own brands, supply chains and the transformation of the agrofood system. In: International Journal of the Sociology of Agriculture and Food 13(1): 1-8.

Burgmann, Verity/Burgmann, Meredith (1999): 'A rare shift in public thinking': Jack Mundey and the New South Wales Builders Labourers' Federation. In: Labour History 77: 44-63.

Burkett, Paul (1999): Marx and Nature. A Red and Green Perspective. New York.

Butollo, Florian/ten Brink, Tobias (2012): Challenging the Atomization of Discontent. In: Critical Asian Studies 44:(3) 419-440.

Cari, Sara/Domínguez, Rafael (2016): Ecuador's Buen Vivir. A New Ideology for Development. Latin American Perspectives 43(1): 18-33.

Chattopadhyay, Kunal/Marik, Soma (2016): India on Strike. URL: https://www.jacobinmag. com/2016/10/indian-workers-general-strike/, Zugriff 30.10.2017.

Clapp, Jennifer (2014): Financialization, distance and global food politics. In: The Journal of Peasant Studies 41(5): 797-814.

Coe, Neil M./Dicken, Peter/Hess, Martin (2008): Global Production Networks: Realizing the Potential. In: Journal of Economic Geography 8: 271-295.

Cumbers, Andy/Nativel, Corinne/Routledge, Paul (2008): Labour Agency and Union Positionalities in Global Production Networks. In: Journal of Economic Geography 8: 369-387.

Demaria, Federico/Schneider, Francois/Sekulova, Filka/Martinez-Alier, Joan (2013): What is Degrowth? From an Activist Slogan to a Social Movement. In: Environmental Values 22 (2013): 191-215.

Dicken, Peter (2011): Global Shift. Mapping the Changing Contours of the World Economy. New York.

Dietz, Kristina/Pye, Oliver/Engels, Bettina (2016): Sozial-räumliche Dynamiken der Agrartreibstoffe. Transnationale Netzwerke, skalare Rekonfigurationen, umkämpfte Orte und Territorien. PROKLA 46(3): 423-440.

Dixon, Jane (2009): From the imperial to the empty calorie: how nutrition relations underpin food regime transitions. In: Agriculture and Human Values 26(4): 321-333.

Dörre, Klaus (2013): Landnahme. Triebkräfte, Wirkungen und Grenzen kapitalistischer Wachstumskritik. In: Backhouse, Maria/Gerlach, Olaf/Kalmring, Stefan/Nowak, Andreas (Hg.): Die globale Einhegung. Krise, ursprüngliche Akkumulation und Landnahmen im Kapitalismus. Münster: 112-140.

Dove, Michael (2011): The Banana Tree at the Gate. A History of Marginal Peoples and Global Markets in Borneo. New Haven. 
Foster, John Bellamy (2000): Marx's Ecology. Materialism and Nature. New York.

- (2011): Capitalism and Degrowth - an Impossibility Theorem. URL: https://monthlyreview. org/2011/01/01/capitalism-and-degrowth-an-impossibility-theorem/, Zugriff 22.09.2017.

-/Clark, Brett/York, Richard (2010): The Ecological Rift. Capitalism's War on the Earth. New York.

Görg, Christoph (2004): Postfordistische Transformation der Naturverhältnisse. In: Beerhorst, Joachim u.a.(Hg.): Kritische Theorie im gesellschaftlichen Strukturwandel. Frankfurt/M: 199-226. - (2003): Regulation der Naturverhältnisse. Münster.

Harvey, David (1981): The Spatial Fix - Hegel, von Thunen, and Marx. In: Antipode 13(1): 1-12. - (2003): The New Imperialism. Oxford.

HBS und BUND (2016): Fleischatlas. URL: https://www.bund.net/fileadmin/user_upload bund/publikationen/massentierhaltung/massentierhaltung_fleischatlas_regional_2016.pdf, Zugriff: 7.11.2017.

Holloway, John (2005): Change the World Without Taking Power. London.

Juliawan, Benny Hari (2011): Street-level Politics: Labour Protests in Post-authoritarian Indonesia. In: Journal of Contemporary Asia 41(3): 349-370.

Leong, Apo (2017): Labour Activism Under the 'New Normal' in China. In: Panimbang, Fahmi (Hg): Resistance on the Continent of Labour. Strategies and Initiatives of Labour Organizing in Asia. Hongkong: 41-54.

Marx, Karl (1844): Ökonomisch-philosophische Manuskripte. In: Marx-Engels-Werke, Bd. 40. Berlin 1968.

- (1890): Das Kapital. Kritik der politischen Ökonomie. Band 1. In: Marx-Engels-Werke, Bd. 23. Berlin 1969.

McMichael, Philip (2009): A food regime genealogy. In: The Journal of Peasant Studies 36(1): 139-169.

Missingham, Bruce D. (2003): The Assembly of the Poor in Thailand. From Local Struggles to National Protest Movement. Chiangmai.

Moore, Jason (2011): Wall Street is a way of organizing nature. In: Upping the Anti: A Journal of Theory and Action 12: 39-53.

Moore, Jason (2015): Capitalism in the Web of Life. Ecology and the Accumulation of Capital. London.

Mufakhir, Abu (2017): Alliances of Labour Unions as the Backbone of General Strikes in Indonesia. In: Panimbang, Fahmi (Hg): Resistance on the Continent of Labour. Strategies and Initiatives of Labour Organizing in Asia. Hongkong: 261-288.

Pye, Oliver (2005): Khor Jor Kor - Forest Politics in Thailand. Bangkok.

- (2013): Migration, Netzwerke und Alltagswiderstand: Die umkämpften Räume der Palmölindustrie. Peripherie 33(132): 466-493.

-/Radjawali, Irendra/Julia (2017): Land Grabs and the River. In: Canadian Journal of Development Studies 38(3): 378-394.

-/Daud, Ramlah/Tatat/Harmono, Yuyun (2012): Precarious Lives: Transnational biographies of migrant oil palm workers, Asia Pacific Viewpoint 53(3): 330-342.

Radjawali, Irendra/Pye, Oliver/Flitner, Michael (2017): Recognition through reconnaissance? Using drones for counter-mapping in Indonesia. In: The Journal of Peasant Studies 44(4): 817-833.

Siu, Kaxton/Chan, Anita (2015): Strike Wave in Vietnam, 2006-2011. In:Journal of Contemporary Asia 45(1): 71-91.

Vebu (ohne Datum): Anzahl der Veganer und Vegetarier in Deutschland. URL: https://vebu.de/ veggie-fakten/entwicklung-in-zahlen/anzahl-veganer-und-vegetarier-in-deutschland/,Zugriff: 7.11.2017. 